\title{
Stability analysis of a host parasite model
}

\author{
Ünal Ufuktepe ${ }^{*}$ and Sinan Kapçak
}

\section{${ }^{*}$ Correspondence:}

unal.ufuktepe@ieu.edu.tr

Department of Mathematics, İzmir

University of Economics, Balcova,

Izmir, Turkey

\begin{abstract}
Host parasite models are similar to host parasitoid models except that the parasite does not necessarily kill the host. Leslie/Gower model (Leslie and Gower in Biometrika 47(3/4):219-234, 1960) played a historical role in ecology. We consider the stability of Misra and Mitra's model (Misra and Mitra in Comput. Math. Appl. 52:525-538, 2006). We study this system analytically and improve the results of Misra and Mitra (Comput. Math. Appl. 52:525-538, 2006).
\end{abstract}

MSC: 39A11;92D25

Keywords: host-parasite; predator-prey; Beddington model; discrete dynamical systems

\section{Introduction}

Interaction (predator-prey, host-parasite, host-parasitoid, mutualism, competition) models of two species in nature, say

$$
\begin{aligned}
& x_{t+1}=f\left(x_{t}, y_{t}\right), \\
& y_{t+1}=g\left(x_{t}, y_{t}\right),
\end{aligned}
$$

in general describe the change in the populations (i) by assuming a model of growth for both species in the absence of the other, and (ii) by describing the effect of their interaction on each other. The simplest model is

$$
\begin{aligned}
& x_{t+1}=a x_{t}+b x_{t} y_{t}, \\
& y_{t+1}=c y_{t}+d x_{t} y_{t} .
\end{aligned}
$$

For example, choosing $a>1, b<0,0<c<1, d>0$ will give us a predator-prey model where the growth or decline is exponential in the absence of the other species in both cases ( $x_{t}$ is the prey population). Choosing $a, c>1$ and $b, d>0$ gives rise to mutualism, where both species get a boost from living together. The mass-action terms $x_{t} y_{t}$ indicate random encounters between the species with a significant outcome (eating or infecting one another, etc.). One of the earliest realistic models of two-species interaction was developed by Nicholson and Bailey, who applied it to the parasitoid Encarsia formosa and the host Trialeurodes vaporariorum. A parasitoid is a parasite that can live on its own as an adult, which then lays eggs into a host, eventually causing its death. The general host-

(C) 2013 Ufuktepe and Kapçak; licensee Springer. This is an Open Access article distributed under the terms of the Creative Commons Attribution License (http://creativecommons.org/licenses/by/2.0), which permits unrestricted use, distribution, and reproduction in any medium, provided the original work is properly cited. 
parasitoid model is

$$
\begin{aligned}
& H_{t+1}=r H_{t} f\left(H_{t}, P_{t}\right), \\
& P_{t+1}=e H_{t}\left(1-f\left(H_{t}, P_{t}\right)\right),
\end{aligned}
$$

where $f\left(H_{t}, P_{t}\right)$ is the fraction of host population that is not parasitized at time $t, r$ is the fecundity of the host, and $e$ is that of the parasite, that is, the average number of successful eggs laid per individual. Note that the parasitoids die in the absence of the host, and for this reason, they have been successfully used for eradicating insect pests. In the NicholsonBailey model, the function $f$ is determined, under the assumptions of the mass-action principle and Poisson distribution of the number of encounters, to be $f\left(H_{t}, P_{t}\right)=e^{-c P_{t}}$ (the positive constant $c$ is called the 'searching efficiency' of the parasitoid).

Cooperation between the two populations is reflected by the fact that the transition function for each population is an increasing function of the other population size. There are several papers on the two-dimensional systems of difference equations with rational nonlinearities. Most of these papers are models for either competition, as in [1-3], or cooperation, as in [4-7]. Consider the following system of difference equations with parameters $A, B>0$ and initial values $x_{0}, y_{0} \geq 0$.

$$
\begin{aligned}
x_{n+1} & =\frac{A x_{n} y_{n}}{1+y_{n}}, \\
y_{n+1} & =\frac{B y_{n} x_{n}}{1+x_{n}} .
\end{aligned}
$$

This system represents the rule by which two discrete, cooperating populations reproduce from one generation to the next. The phase variables $x_{n}$ and $y_{n}$ denote population sizes during the $n$th generation and the sequence or orbit $\left\{\left(x_{n}, y_{n}\right): n=0,1,2, \ldots\right\}$ depicts how the populations evolve over time [3].

In Hassell's model [2], the growth factor is of the form

$$
F\left(H_{t}\right)=\frac{R}{\left(1+a H_{t}\right)^{b}},
$$

where $a, b>0$. This is the population model we will assume for our host species. Hassell et al. [2] collected $R$ and $b$ values for about two dozen species from field and laboratory observations and noted that the majority of these cases were within the stable region.

In this paper we study the following host-parasite model, which was studied by Misra and Mitra [8], where the growing host is infected with the parasite:

$$
\begin{aligned}
& H_{t+1}=\frac{R H_{t}}{\left(1+H_{t}\right)^{b}} e^{-c P_{t}}, \\
& P_{t+1}=H_{t}\left(1-e^{-c P_{t}}\right) .
\end{aligned}
$$

Note that such simplifications, including the convention $a=1$ in the Hassell model, lead to the interpretation of the 'population' variable as a 'suitable multiple of the population. 


\section{Fixed points of the system (1.4)}

In order to find the fixed points $\left(H^{*}, P^{*}\right)$ of the system (1.4), we set $H_{t}=H_{t+1}=H^{*}$ and $P_{t}=P_{t+1}=P^{*}$ :

$$
\begin{aligned}
& H^{*}=\frac{R H^{*}}{\left(1+H^{*}\right)^{b}} e^{-c P^{*},} \\
& P^{*}=H^{*}\left(1-e^{-c P^{*}}\right) .
\end{aligned}
$$

We first observe that for $H^{\prime \prime}=0$, we have the extinction fixed point $(0,0)$ for any values of parameters. For $H^{\prime \prime} \neq 0$ and $P^{*}=0$, we obtain the solution $\left(R^{\frac{1}{b}}-1,0\right)$. For $H^{*} \neq 0$ and $P^{*} \neq 0$, from the first equation in (1.4), we have

$$
P^{*}=\frac{1}{c} \ln \left[\frac{R}{\left(1+H^{*}\right)^{b}}\right]
$$

Now, we can see that the parameter $R$ is important for the existence of the fixed points other than the extinction fixed point $(0,0)$. We have the following cases.

Case 1: $R \leq 1$.

For this case, we have $R^{\frac{1}{b}}-1 \leq 0$ and $\frac{1}{c} \ln \left[\frac{R}{\left(1+H^{3}\right)^{b}}\right]<0$. Hence, there is no exclusion and coexistence fixed point for $R<1$.

Case 2: $R>1$.

By using the first equation in (2.1), assuming that $H^{*} \neq 0$, we have

$$
e^{-c P^{*}}=\frac{\left(1+H^{*}\right)^{b}}{R} \text {. }
$$

Combining equation (2.3) and the second equation in (2.1), we obtain

$$
P^{*}=H^{*}\left(1-\frac{\left(1+H^{*}\right)^{b}}{R}\right)
$$

Now, we can write the first equation in (1.4) as

$$
H^{*}=\frac{R H^{*}}{\left(1+H^{*}\right)^{b}} e^{-c H^{*}\left(1-\frac{\left(1+H^{*}\right)^{b}}{R}\right)}
$$

or

$$
\left(1+H^{*}\right)^{b} e^{c H^{*}-\frac{c}{R} H^{*}\left(1+H^{*}\right)^{b}}=R
$$

an equation in the variable $H^{*}$. Let us denote

$$
z=F(x)=(1+x)^{b} e^{c x-\frac{c}{R} x(1+x)^{b}} .
$$

When the graph of $F$ intersects the horizontal line $z=R$, some fixed points are obtained. Note that $x=R^{\frac{1}{b}}-1$ is a solution of the equation $F(x)=R$, which corresponds to the fixed point $\left(R^{\frac{1}{b}}-1,0\right)$ of the system (1.4). We investigate if there exist some other intersection points. 


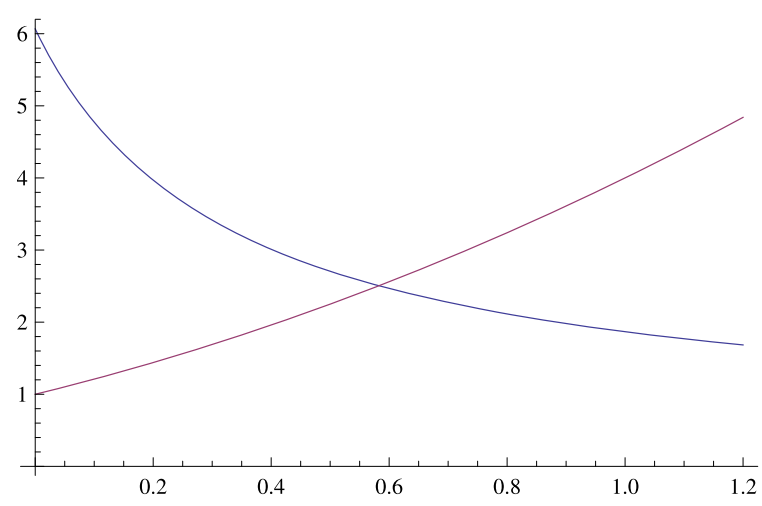

Figure 1 The intersection point is the point where $F^{\prime}(x)=0$.

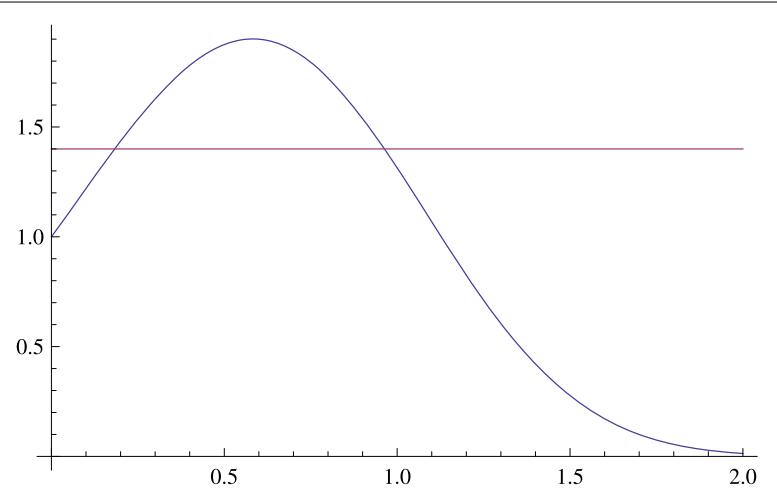

Figure $2 z=F(x), z=R$ where $b=2, c=0.6, R=1.4$

Setting $F^{\prime}(x)=0$, we obtain the equation

$$
\frac{R(b+c+c x)}{c(1+x+b x)}=(1+x)^{b} .
$$

For $x>0$, the function on the right-hand side has $y$-intercept 1 and is monotonically increasing without bound. On the other hand, the function on the left-hand side is monotonically decreasing, has $y$-intercept $R(1+b / c)>1$, and converges to $R /(1+b)$ as $x \rightarrow \infty$. Thus, there is a unique intersection point which means there exists only one critical point (see Figure 1).

Since $F(0)=1, F^{\prime}(0)=\frac{c(R-1)+b R}{R}>0, F(x) \rightarrow 0$ as $x \rightarrow \infty$, the critical point is a local maximum. See Figure 2.

In Figure 2, we know that one of the intersection points is the solution $x=R^{\frac{1}{b}}-1$. The other intersection point, say $\bar{H}$, may or may not be a component of a positive fixed point. In order to guarantee that the $P$ component, say $\bar{P}$, is also positive, we solve $P^{*}>0$ in equation (2.2) and find

$$
H^{*}<R^{\frac{1}{b}}-1
$$


Thus, there exists a positive fixed point only if the $H$ component of it must be less than the number $R^{\frac{1}{b}}-1$, which means among the two intersection points, the one on the right must be $R^{\frac{1}{b}}-1$ and hence $F^{\prime}\left(R^{\frac{1}{b}}-1\right)<0$. Solving this inequality, we obtain the condition for the existence of the positive fixed point: $R>\left(1+\frac{1}{c}\right)^{b}$.

Thus, we obtain the following result.

Theorem 2.1 For the system (1.4), the following statements hold true.

a. If $R \leq 1$, then the only fixed point is the extinction fixed point $(0,0)$.

b. If

$$
1<R \leq\left(1+\frac{1}{c}\right)^{b}
$$

then there exist two fixed points: the extinction fixed point $(0,0)$ and the exclusion fixed point $\left(R^{\frac{1}{b}}-1,0\right)$.

c. If

$$
R>\left(1+\frac{1}{c}\right)^{b}
$$

then there exist three fixed points: extinction fixed point $(0,0)$, exclusion fixed point $\left(R^{\frac{1}{b}}-1,0\right)$, and a coexistence fixed point.

\section{Stability analysis of the system (1.4)}

In this section, the stability of the fixed points is examined.

The Jacobian matrix of the system (1.4) is

$$
J=\left(\begin{array}{cc}
R(1+H-b H)(1+H)^{-1-b} e^{-c P} & -c R H(1+H)^{-b} e^{-c P} \\
1-e^{-c P} & c H e^{-c P}
\end{array}\right) .
$$

At $(0,0)$, the Jacobian becomes

$$
J_{0}=\left(\begin{array}{ll}
R & 0 \\
0 & 0
\end{array}\right) .
$$

The eigenvalues for the fixed point $(0,0)$ are $\lambda_{1}=R$ and $\lambda_{2}=0$. Hence, $(0,0)$ is asymptotically stable if $R<1$. We now consider the exclusion fixed point.

Theorem 3.1 For the system (1.4), the exclusion fixed point $\left(R^{\frac{1}{b}}-1,0\right)$ is asymptotically stable if

$$
\max \left(\frac{c}{c+1}, \frac{b-2}{b}\right)<R^{-\frac{1}{b}}<1 .
$$

Proof The Jacobian matrix evaluated at this point is given by

$$
J_{2}=\left(\begin{array}{cc}
1+b\left(-1+R^{-\frac{1}{b}}\right) & -c\left(-1+R^{\frac{1}{b}}\right) \\
0 & c\left(-1+R^{\frac{1}{b}}\right)
\end{array}\right),
$$

where the eigenvalues are $\lambda_{1}=1+b\left(-1+R^{-\frac{1}{b}}\right)$ and $\lambda_{2}=c\left(-1+R^{\frac{1}{b}}\right)$. Applying the stability conditions $\left|\lambda_{1}\right|<1$ and $\left|\lambda_{2}\right|<1$, we obtain the desired result. 
Note that the condition $\left|\lambda_{2}\right|<1$ yields $R<\left(1+\frac{1}{c}\right)^{b}$, for which there does not exist any coexistence fixed point. When the coexistence fixed point appears, the exclusion fixed point loses stability. We confirm our result by visual representation of the system for some values of parameters: Taking $b=1.15, c=2.2, R=1.5$, for which the condition in Theorem 3.1 is satisfied, the exclusion fixed point is locally asymptotically stable (see Figure 3 ). However, taking $c=3.2$ and leaving the other parameters as they are, the coexistence fixed point appears and the exclusion fixed point loses stability (see Figure 4).
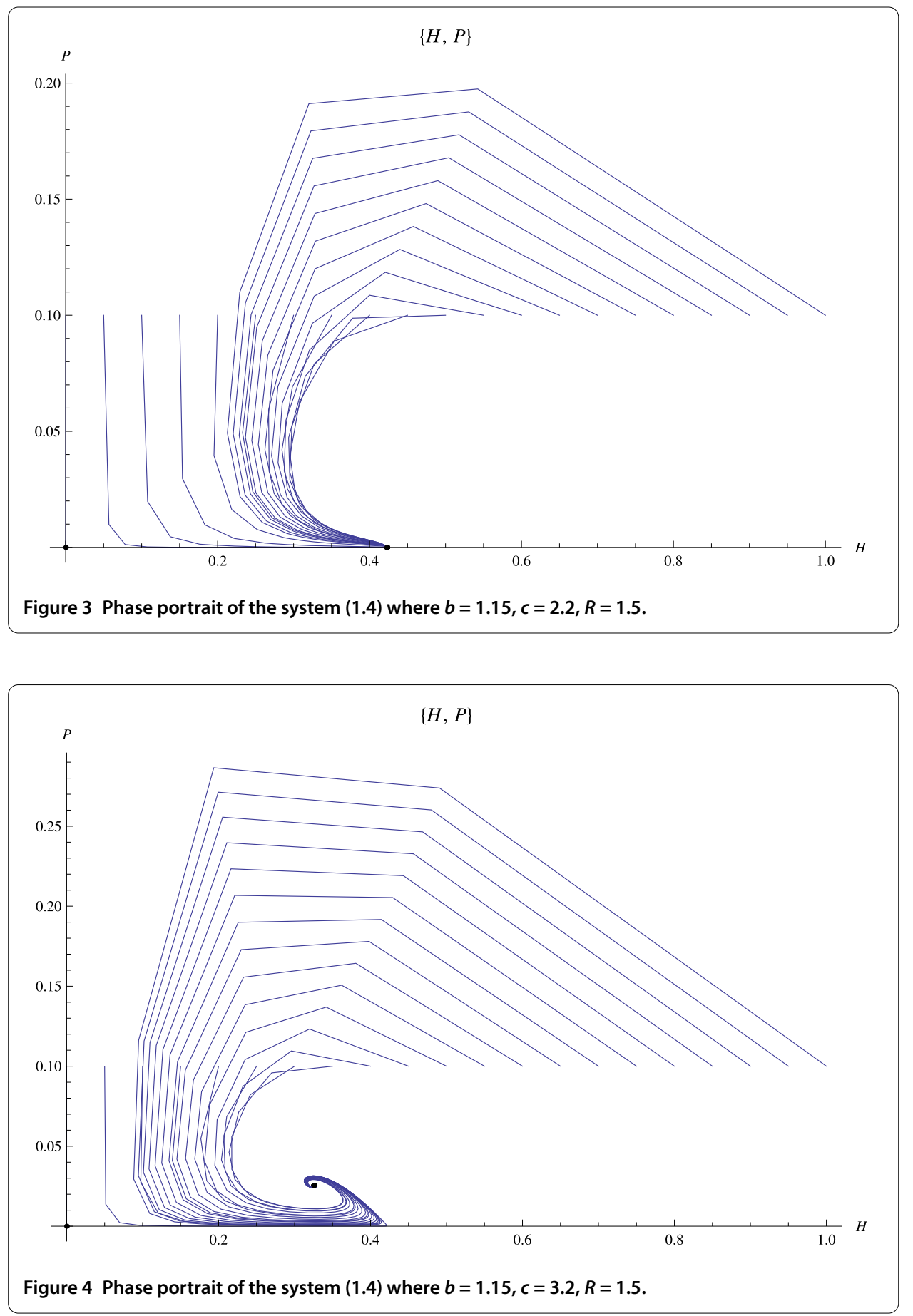
Competing interests

The authors declare that they have no competing interests.

\section{Authors' contributions}

UU and SK studied together on all steps of the main problem. SK wrote the Mathematica codes for the phase potrait of the system. All authors read and approved the final manuscript.

\section{Acknowledgements}

Dedicated to Professor Hari M Srivastava.

\section{Received: 15 November 2012 Accepted: 8 March 2013 Published: 28 March 2013}

\section{References}

1. Clark, D, Kulenovik, MRS: A coupled system of rational difference equations. Comput. Math. Appl. 43, 849-867 (2002)

2. Hassell, MP, Comins, HN: Discrete time models for two-species competition. Theor. Popul. Biol. 9, $202-221$ (1976)

3. Kulenovic, MRS, Nurkanovic, M: Global asymptotic behavior of a two dimensional system of difference equations modeling cooperation. J. Differ. Equ. Appl. 9(1), 149-159 (2003)

4. Elaydi, S: An Introduction to Difference Equations. Springer, Berlin (2000)

5. Elaydi, S: Discrete Chaos: With Applications in Science and Engineering, 2nd edn. Chapman \& Hall/CRC, Boca Raton (2008)

6. Selgrade, JF, Ziehe, M: Convergence to equilibrium in a genetic model with differential viability between the sexes. J. Math. Biol. 25, 477-490 (1987)

7. Smith, HL: Monotone Dynamical Systems: An Introduction to the Theory of Competitive and Cooperative Systems. Am. Math. Soc., Providence (1995)

8. Misra, JC, Mitra, A: Instabilities in single-species and host-parasite systems: period-doubling bifurcations and chaos. Comput. Math. Appl. 52, 525-538 (2006)

doi:10.1186/1687-1847-2013-79

Cite this article as: Ufuktepe and Kapçak: Stability analysis of a host parasite model. Advances in Difference Equations 2013 2013:79.

\section{Submit your manuscript to a SpringerOpen ${ }^{\ominus}$ journal and benefit from:}

- Convenient online submission

- Rigorous peer review

- Immediate publication on acceptance

- Open access: articles freely available online

- High visibility within the field

- Retaining the copyright to your article 